\title{
Increased risk of cardiovascular events and mortality among non-diabetic chronic kidney disease patients with hypertensive nephropathy: the Gonryo study
}

\author{
Masaaki Nakayama ${ }^{1,2}$, Toshinobu Sato ${ }^{3}$, Mariko Miyazaki ${ }^{1}$, Masato Matsushima ${ }^{1,4}$, Hiroshi Sato ${ }^{1,5}$, \\ Yoshio Taguma ${ }^{1,3}$ and Sadayoshi Ito ${ }^{1}$
}

To examine the clinical significance of hypertensive nephropathy (HN) among non-diabetic chronic kidney disease (CKD) patients. The study comprised 2692 CKD patients recruited from 11 outpatient nephrology clinics; these included 1306 patients with primary renal disease (PRD), 458 patients with HN, 283 patients with diabetic nephropathy (DN) and 645 patients with other nephropathies (ONs). All patients fulfilled the criteria of CKD, with a persistent low estimated glomerular filtration rate (eGFR) $<60 \mathrm{ml} \mathrm{min}-1$ per $1.73 \mathrm{~m}^{2}$ or proteinuria as determined by a urine dipstick test. The risk factors for cardiovascular disease (CVD), such as ischemic heart disease, congestive heart failure and stroke; all-cause mortality; and progression to end-stage renal failure (dialysis induction) were analyzed using a Cox proportional hazards model in each group. During a mean follow-up period of 22.6 months from recruitment, 100 patients were lost to follow-up and 192 patients began chronic dialysis therapy. A total of 115 CVD events occurred (stroke in 37 cases), and 44 patients died. Regarding CVD events and death, there were significant differences in the hazard ratios (HRs) for the groups of patients with different underlying renal diseases as determined by both univariate and multivariate analysis adjusted for confounding factors including estimated glomerular filtration rate: PRD, 1.0 (reference); HN, 3.33 (95\% confidence interval, 1.82-6.09); DN, 5.93 (2.80-12.52); and ON, $2.22(1.22-4.05)$. However, there were no differences in the hazard ratio for dialysis induction for the groups of patients with different underlying renal diseases. HN is associated with an increased risk of CVD events and death among non-diabetic CKD patients, which highlights the clinical significance of $\mathrm{HN}$.

Hypertension Research (2011) 34, 1106-1110; doi:10.1038/hr.2011.96; published online 28 July 2011

Keywords: cardiovascular disease; chronic kidney disease; hypertensive nephropathy

\section{INTRODUCTION}

Chronic kidney disease $(\mathrm{CKD})^{1}$ is a well-known independent risk factor for cardiovascular disease (CVD), including stroke, progression to end-stage renal failure and all-cause mortality in the general population. $^{2-8}$

The relation between excess CVD morbidity and mortality, and decreased kidney function has been well demonstrated in diabetic patients ${ }^{9,10}$ in specific sub-populations with preexisting heart disease, ${ }^{11,12}$ hypertension ${ }^{13}$ and dislipidemia, ${ }^{14}$ and in the elderly. ${ }^{15}$

Patients with primary or secondary kidney diseases are exposed to several unique factors that increase the frequency of CVD events. These factors include hyperlipidemia and coagulopathy due to nephritic syndrome, systemic inflammation-associated vasculitides, underlying collagen or infectious disease, and the use of therapeutic agents such as steroids. ${ }^{16-18}$ Even though patients with hypertensive nephropathy (HN, nephrosclerosis) are believed to be at high risk for progression to kidney failure, ${ }^{19}$ the impact of $\mathrm{HN}$ on the frequency of CVD events compared with the impact of other nephropathies (ONs) has not been clearly demonstrated.

Accordingly, in terms of risk stratification of patients, it is crucially important to clarify the clinical outcomes of CKD with respect to the underlying renal diseases, especially for CKD cases that are not the result of diabetes. Only a few reports have examined this issue, including our preliminary report. ${ }^{20-22}$

The present study aimed to address this issue in a cohort of patients from nephrology clinics.

\section{METHODS}

Study population (Gonryo CKD cohort)

The Gonryo CKD project is a prospective survey of the patient characteristics and outcomes of individuals who visit outpatient nephrology clinics in the Miyagi Prefecture (Northeast area of Japan), the details of which have been

${ }^{1}$ Tohoku University Graduate School of Medicine, Center for Advanced Integrated Renal Science, Sendai, Japan; ${ }^{2}$ Fukushima Medical University School of Medicine, Fukushima, Japan; ${ }^{3}$ Sendai Shakaihoken Hospital, Kidney Center, Sendai, Japan; ${ }^{4}$ The Jikei University School of Medicine, Department of Clinical Research, Tokyo, Japan and ${ }^{5}$ Tohoku University Graduate School of Pharmacology, Department of Clinical Pharmacology, Sendai, Japan

Correspondence: Dr M Nakayama, Tohoku University Graduate School of Medicine, Center for Advanced Integrated Renal Science, 1-1 Seiryo-machi, Aoba-ku, Sendai 980-8574, Japan. 
reported elsewhere. ${ }^{22}$ Eleven affiliated hospitals with Tohoku University, including one university hospital (Tohoku University Hospital), are participating in the project. Patient registration was originally requested for all patients who provided informed consent for participation in the project. The study protocol was approved by the institutional review board of the Tohoku University School of Medicine and by the respective participating hospitals.

Registration was conducted from May 2006 to November 2008, and 4015 patients were registered. Among the original registered patients, certain subjects were excluded from the present analysis-150 cases lacking data on serum creatinine levels and 241 cases with unknown underlying renal diseases. Among patients with essential hypertension and estimated glomerular filtration rates (eGFRs) above $60 \mathrm{ml} \mathrm{min}^{-1}$ per $1.73 \mathrm{~m}^{2}$, those who did not have positive proteinuria findings at registration $(n=836)$ and those lacking urinary testing results $(n=96)$ were excluded. As a result, 2692 patients with complete CKD criteria were selected ${ }^{1}$ and were subjected to analysis.

\section{Patient classification and primary outcomes}

Patients were classified according to one of four underlying renal diseases diagnosed by the attending physicians at the participating hospitals (Table 1): primary renal disease (PRD), defined by primary glomerulonephritis and tubulointerstitial nephritis, including biopsy-proven cases (81\%); HN, defined by a history of hypertension and the absence of other possible disorders, including cases of biopsy-proven nephrosclerosis (20.8\%); diabetic nephrop- athy, defined by a history of diabetes accompanying nephropathy and the absence of other possible renal disorders or presenting with nephropathy with diabetic retinopathy and the absence of other possible renal disorders, including biopsy-proven diabetic nephropathy (24.9\%); and ONs, defined by ONs not included in the other three groups, including biopsy-proven cases (24.9\%). The $\mathrm{HN}$ cases included in the present classification were in those patients who had an eGFR below $60 \mathrm{ml} \mathrm{min}^{-1}$ per $1.73 \mathrm{~m}^{2}$ or positive proteinuria as determined by a dipstick test.

The primary outcomes of this survey included CVD events, such as angina pectoris, acute myocardial infarction, congestive heart failure, stroke (cerebral bleeding and infarction), and all-cause death before commencement of chronic dialysis therapy. Outcomes within 12 months after registration were surveyed using the medical records of the hospitals, death certificates and interviews with attending physicians at the time of annual checkups. An episode of CVD was defined as disease of the circulatory system (International Classification of Disease, 10th revision: I00 to I99), and the number of patients with angina pectoris or acute myocardial infarction included those who had received coronary stenting, angioplasty or bypass surgery, or who had a definite clinical course of acute myocardial infarction. In patients with congestive heart failure, only those who were admitted for treatment were counted. Diagnosis of stroke and stroke subtypes was based on the Classification of Cerebrovascular Diseases III by the National Institute of Neurological Disorders and Stroke, ${ }^{23}$ and only cases confirmed by computed tomography or magnetic resonance imaging of the brain were counted.

Table 1 Patient characteristics

\begin{tabular}{|c|c|c|c|c|c|}
\hline & $A / l$ & $P R D$ & ONs & $H N$ & $D N$ \\
\hline$n$ & 2.694 & 1.306 & 643 & 462 & 283 \\
\hline Age (years) & $60.0 \pm 16.2$ & $55.7 \pm 16.6$ & $58.6 \pm 15.7$ & $70.3 \pm 11.4$ & $66.5 \pm 12.6$ \\
\hline Gender (male) & $1441(53.5 \%)$ & $716(54.8 \%)$ & 275 (42.8\%) & 262 (56.7\%) & $188(66.4 \%)$ \\
\hline $\mathrm{BMI}$ & $23.5 \pm 3.8$ & $23.4 \pm 3.8$ & $22.9 \pm 3.7$ & $24.2 \pm 3.8$ & $24.1 \pm 3.8$ \\
\hline \multicolumn{6}{|l|}{ Blood pressure $(\mathrm{mmHg})$} \\
\hline Systolic & $130.95 \pm 16.2$ & $129.21 \pm 15.1$ & $129.33 \pm 15.8$ & $134.68 \pm 17.4$ & $136.64 \pm 17.5$ \\
\hline Diastolic & $76.7 \pm 10.9$ & $77.3 \pm 10.4$ & $76.7 \pm 10.7$ & $76.4 \pm 11.8$ & $74.0 \pm 11.6$ \\
\hline \multicolumn{6}{|l|}{ CKD stage (\%) } \\
\hline Stage $1+2$ & 40.3 & 49.1 & 47.4 & 17.7 & 20.4 \\
\hline Stage 3 & 37.6 & 35.7 & 31.1 & 57.6 & 28.3 \\
\hline Stage 4 & 13.4 & 10.3 & 14.5 & 15.8 & 21.6 \\
\hline Stage 5 & 8.7 & 4.9 & 7.0 & 8.9 & 29.7 \\
\hline \multicolumn{6}{|l|}{ Comorbidities (\%) } \\
\hline Cardiac disease & 12.8 & 7.5 & 12.3 & 21.2 & 24.7 \\
\hline Stroke & 6.5 & 3.5 & 5.9 & 11.9 & 12.7 \\
\hline Diabetes & 27.4 & 15.5 & 18.0 & 34.2 & 100.0 \\
\hline Hypertension & 77.1 & 72.8 & 70.6 & 93.7 & 89.4 \\
\hline Hyperlipidemia & 42.6 & 44.6 & 36.8 & 42.2 & 51.6 \\
\hline \multicolumn{6}{|l|}{ Pharmacotherapy (\%) } \\
\hline ARB/ACEI & 62.7 & 62.1 & 52.6 & 70.1 & 76.3 \\
\hline Statin & 34.7 & 36.1 & 30.0 & 32.3 & 43.1 \\
\hline ESA & 6.5 & 3.6 & 4.8 & 7.8 & 21.9 \\
\hline Steroid & 25.3 & 32.9 & 36.2 & 2.8 & 2.1 \\
\hline Proteinuria (\%) & 49.6 & 47.3 & 41.2 & 49.8 & 78.9 \\
\hline Hemoglobin (g dl-1) & $12.8 \pm 2.1$ & $13.2 \pm 1.9$ & $12.6 \pm 2.0$ & $12.7 \pm 2.1$ & $11.6 \pm 2.3$ \\
\hline Total cholesterol (mg dl ${ }^{-1}$ ) & $197.6 \pm 38.7$ & $198.7 \pm 35.9$ & $203.5 \pm 41.3$ & $190.99 \pm 39.9$ & $190.4 \pm 41.4$ \\
\hline Smoker (\%) & 16.2 & 15.8 & 14.6 & 16.2 & 21.6 \\
\hline Renal biopsy proven (\%) & 62.7 & 81.0 & 53.5 & 20.8 & $\begin{array}{c}24.9 \\
\text { mean } \pm \text { s.d. }\end{array}$ \\
\hline
\end{tabular}




\section{Data collection}

Serum creatinine levels were measured using the enzyme assay method. Kidney function was determined using the formula for eGFR for Japanese individuals. ${ }^{24}$ Positive results for urinary protein were identified using the dipstick test for spot urine or an autoanalyzer. Patients were considered to be positive for macroalbuminuria when the dipstick result was positive or greater, corresponding to a urinary protein level $>30 \mathrm{mg} \mathrm{dl}^{-1} .{ }^{25}$ Blood pressure was measured at local medical centers in outpatient clinics using an automatic sphygmomanometer based on the Korotkoff sound technique with the subject in a seated position. Information on medications at baseline and each patient's history of CVD, diabetes mellitus, hypertension and hyperuricemia were obtained from the medical records or from the results of blood examinations at registration. Subjects receiving lipid-lowering drugs or displaying serum cholesterol levels $>220 \mathrm{mg} \mathrm{dl}^{-1}$ were considered to have hypercholesterolemia. Subjects with fasting glucose levels $>126 \mathrm{mg} \mathrm{dl}^{-1}$ or non-fasting glucose levels $>200 \mathrm{mg} \mathrm{dl}^{-1}$ or who used insulin or oral antihyperglycemic drugs were defined as having diabetes mellitus.

\section{Data analysis}

Associations between primary outcomes and either baseline kidney function or underlying renal disease were examined using Cox proportional hazard model analysis adjusted for confounding factors.

Data are shown as means \pm s.d. A $P$-value $<0.05$ indicated statistical significance. All statistical analyses were conducted using STATA version 10.0 software (StataCorp LP, College Station, TX, USA).

\section{RESULTS}

During an observation period of $22.6 \pm 11.9$ months, 100 patients were lost because of a switch to other medical services or to the patient quitting due to social reasons, and the follow-up of 192 patients was ended because of the initiation of maintenance dialysis therapy. There were 115 cases of CVD events ( 37 cases of stroke) and 44 cases of allcause death (Table 2).

Table 2 Number of events

\begin{tabular}{|c|c|c|c|c|}
\hline CKD stage & $C V D$ & Stroke & Death & $E S R D$ \\
\hline \multicolumn{5}{|c|}{ Primary renal disease } \\
\hline CKD1+2 & 2 & 1 & 1 & - \\
\hline CKD3 & 7 & 2 & 6 & 2 \\
\hline CKD4 & 2 & 1 & 2 & 8 \\
\hline CKD5 & - & - & 1 & 40 \\
\hline \multicolumn{5}{|c|}{ Hypertensive nephropathy } \\
\hline $\mathrm{CKD} 1+2$ & 4 & - & 1 & 1 \\
\hline CKD3 & 12 & 8 & 6 & - \\
\hline CKD4 & 7 & 4 & 3 & 6 \\
\hline CKD5 & 3 & 2 & 3 & 24 \\
\hline \multicolumn{5}{|c|}{ Diabetic nephropathy } \\
\hline CKD1+2 & 4 & 3 & - & 1 \\
\hline CKD3 & 7 & 1 & 3 & 1 \\
\hline CKD4 & 8 & - & 5 & 18 \\
\hline CKD5 & 10 & 3 & 4 & 54 \\
\hline \multicolumn{5}{|c|}{ Other nephropathies } \\
\hline $\mathrm{CKD} 1+2$ & 2 & 7 & 2 & - \\
\hline CKD3 & 3 & 4 & 3 & 3 \\
\hline CKD4 & 6 & 1 & 4 & 9 \\
\hline CKD5 & 1 & - & - & 25 \\
\hline
\end{tabular}

Abbreviations: CKD, chronic kidney disease; CVD, cardiovascular disease; ESRD, end-stage renal disease (dialysis induction).
In terms of CVD events and all-cause mortality, significant increases in hazard ratios were seen with increasing CKD stage using univariate analysis (Figure 1); however, these trends disappeared after multivariate adjustment (Table 3a). Significant differences in hazard ratios were seen with respect to underlying renal diseases using univariate analysis, and these differences were significant even after adjusting for confounding factors including eGFR (Table 3b).

Dialysis was started only for those patients who had a CKD stage 4 to 5 at the time of entry (Figure 2; CKD1+2: $0.2 \%$, CKD3: $0.6 \%$,

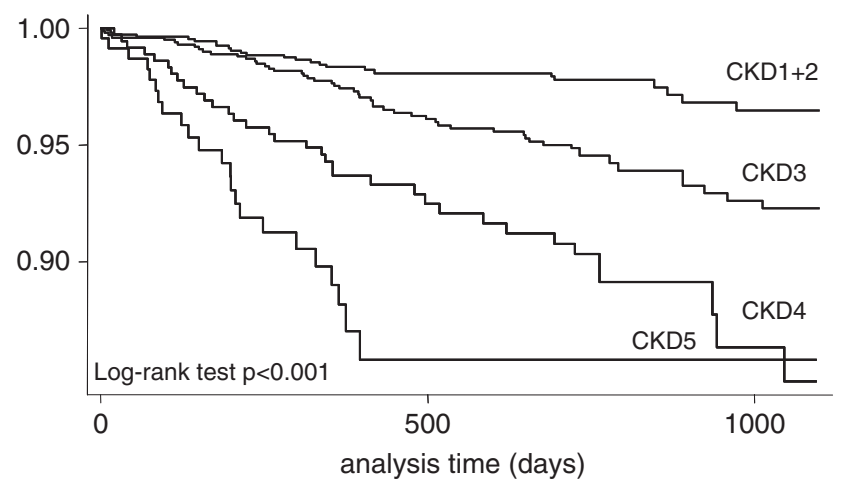

Figure 1 Event-free survival for cardiac disease, apoplexy and all cause of death for patients at different chronic kidney disease (CKD) stages.

Table 3a Risk for endpoints of CVD, stroke and death by CKD stage in all patients

\begin{tabular}{|c|c|c|c|c|c|c|c|}
\hline \multirow[b]{2}{*}{ CKD stage } & \multirow[b]{2}{*}{$C V D$} & \multirow[b]{2}{*}{ Stroke } & \multirow[b]{2}{*}{ Death } & \multicolumn{2}{|c|}{ Univariate analysis } & \multicolumn{2}{|c|}{ Multivariate analysis ${ }^{a}$} \\
\hline & & & & $H R$ & $95 \% \mathrm{Cl}$ & $H R$ & $95 \% \mathrm{Cl}$ \\
\hline CKD $1+2$ & 12 & 11 & 4 & 1.00 & & 1.00 & \\
\hline CKD 3 & 29 & 15 & 18 & 2.21 & $1.37-3.55$ & 1.06 & $0.64-1.77$ \\
\hline CKD 4 & 23 & 6 & 14 & 4.39 & $2.62-7.36$ & 1.76 & $1.00-3.12$ \\
\hline CKD 5 & 14 & 5 & 8 & 7.47 & $4.22-13.24$ & 2.29 & $1.17-4.49$ \\
\hline
\end{tabular}

Abbreviations: $\mathrm{Cl}$, confidence interval; $\mathrm{CKD}$, chronic kidney disease; CVD, cardiovascular disease (such as angina pectoris, acute myocardial infarction and congestive heart failure); HR, hazard ratio.

Adjusted for age, gender, hemoglobin, positive for proteinuria, systolic blood pressure, body mass index, presence of hyperlipidemia, presence of diabetes, prescription of steroid, smoker, history of cardiovascular disease or stroke, use of RAS (renin-angiotensin system) inhibitors.

Table 3b Risk for endpoints of CVD, stroke and death by underlying renal diseases in all patients

\begin{tabular}{|c|c|c|c|c|c|c|c|}
\hline \multirow{2}{*}{$\begin{array}{l}\text { Underlying } \\
\text { renal disease }\end{array}$} & \multirow{2}{*}{$C V D$} & \multirow[b]{2}{*}{ Stroke } & \multirow[b]{2}{*}{ Death } & \multicolumn{2}{|c|}{ Univariate analysis } & \multicolumn{2}{|c|}{ Multivariate analysis ${ }^{\mathrm{a}}$} \\
\hline & & & & $H R$ & $95 \% \mathrm{Cl}$ & $H R$ & $95 \% \mathrm{Cl}$ \\
\hline PRD & 11 & 4 & 10 & 1.00 & & 1.00 & \\
\hline ONs & 12 & 12 & 9 & 3.17 & $1.78-5.62$ & 2.22 & $1.22-4.05$ \\
\hline $\mathrm{HN}$ & 26 & 14 & 13 & 7.12 & $4.18-12.14$ & 3.33 & $1.82-6.09$ \\
\hline DN & 29 & 7 & 12 & 10.88 & $6.29-18.84$ & 5.93 & $2.80-12.52$ \\
\hline
\end{tabular}

Abbreviations: $\mathrm{Cl}$, confidence interval; $\mathrm{CVD}$, cardiovascular disease; DN, diabetic nephropathy; $\mathrm{HN}$, hypertensive nephropathy; HR, hazard ratio; ONs, other nephropathies; PRD, primary renal disease.

aAdjusted for age, gender, hemoglobin, positive for proteinuria, systolic blood pressure, body mass index, presence of hyperlipidemia, presence of diabetes, prescription of steroid, smoker, history of cardiovascular disease or stroke, use of RAS (renin-angiotensin system) inhibitors and estimated GFR (glomerular filtration rate). 


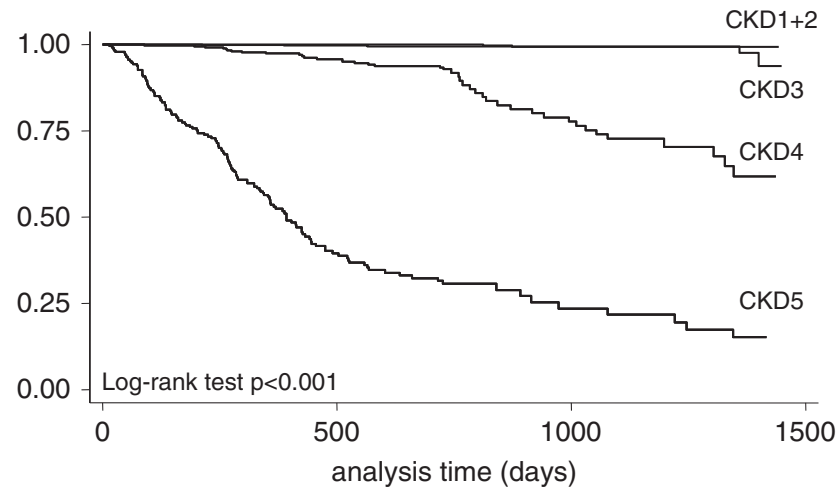

Figure 2 Event-free survival for progression to end-stage renal disease (dialysis induction) for patients at different chronic kidney disease (CKD) stages.

Table 4 Risk of progression to ESRD (dialysis induction) by underlying renal disease

\begin{tabular}{|c|c|c|c|c|c|}
\hline \multirow{2}{*}{$\begin{array}{l}\text { Underlying } \\
\text { Renal disease }\end{array}$} & \multirow[b]{2}{*}{ ESRD } & \multicolumn{2}{|c|}{ Univariate analysis } & \multicolumn{2}{|c|}{ Multivariate analysis ${ }^{a}$} \\
\hline & & $H R$ & $95 \% \mathrm{Cl}$ & $H R$ & $95 \% \mathrm{Cl}$ \\
\hline PRD & 50 & 1.00 & & 1.00 & \\
\hline ONs & 37 & 1.43 & $0.94-2.19$ & 1.11 & $0.70-1.76$ \\
\hline $\mathrm{HN}$ & 31 & 1.09 & $0.70-1.71$ & 1.13 & $0.69-1.88$ \\
\hline DN & 74 & 5.25 & $3.66-7.53$ & 1.25 & $0.68-2.28$ \\
\hline
\end{tabular}

Abbreviations: $\mathrm{Cl}$, confidence interval: $\mathrm{DN}$, diabetic nephropathy. ESRD, end-stage renal disease: $\mathrm{HN}$, hypertensive nephropathy; HR, hazard ratio; ONs, other nephropathies; PRD, primary renal disease.

adjusted for age, gender, hemoglobin, positive for proteinuria, systolic blood pressure, body mass index, presence of hyperlipidemia, presence of diabetes, prescription of steroid, smoker, history of cardiovascular disease or stroke, use of RAS (renin-angiotensin system) inhibitors and estimated GFR (glomerular filtration rate).

CKD4: $11.4 \%$, CKD5: $61.1 \%$ ), and no significant differences were observed with respect to underlying renal diseases after adjusting for confounding factors, including eGFR (Table 4).

\section{DISCUSSION}

This study aimed to clarify the impact of underlying renal diseases on CVD events and death before the initiation of dialysis treatment by analyzing the outcomes of 2692 CKD outpatients from 11 nephrology clinics. After 22.6 months of follow-up, there was a significant difference in the frequencies of CVD events and mortality among groups of patients with different underlying renal diseases, even after adjusting for possible confounding factors including kidney function. These findings showed that patients with $\mathrm{HN}$ represent a high-risk group, except for diabetic nephropathy patients, followed by ONs and PRD. In contrast, in terms of chronic dialysis induction, no significant differences were observed based on underlying renal diseases.

Both traditional and non-traditional mechanisms underlie the increased risk of CVD among CKD patients. Traditional factors include hypertension, diabetes, hyperlipidemia and smoking, whereas non-traditional factors include specific factors related to the uremic milieu, such as fluid overload, calcium/phosphate abnormalities, anemia, malnutrition, enhanced inflammation and oxidative stress, and the accumulation of uremic toxins. ${ }^{26-34}$ Therefore, subjects with vasculopathy demonstrated by traditional factors are thought to undergo accelerated vascular damage along with progression of the CKD stage. Hypertension is a predominant risk factor for CVD in the general population, and it is logical that long-standing exposure to pathological conditions such as hypertension may have resulted in an increased frequency of CVD and mortality among non-diabetic subjects with HN. Several factors could have contributed to the better CVD outcomes in the group with PRD. First, half of the patients with PRD had immunoglobulin A nephropathy; glucocorticoid therapy does not increase the risk of CVD for these patients. ${ }^{34}$ Blood pressure was also more adequately controlled in these patients than in patients in the other groups (Table 1). In addition, prevalent vasculopathy was not predominant in pre-dialysis PRD patients, as has been indicated for pediatric patients. ${ }^{35,36}$ These results indicate that CKD staging cannot be applied on its own to predict which subjects are at high risk of CVD without taking into account the type of underlying renal disease. These results also suggest that individuals with $\mathrm{HN}$ should be the primary targets of CVD prevention measures among non-diabetic CKD patients.

In contrast, the present study revealed that the differences among underlying renal diseases did not have any influence on the frequency of the induction of dialysis after adjusting for confounding factors, including eGFR. In addition, dialysis induction was limited to subjects with CKD5. This result may confirm the clinical notion that CKD5 is the primary criterion for dialysis induction, as recommended in published guidelines. ${ }^{37-39}$

In the present study, several clinical issues that might have biased the analytical results must be considered. First, because all of the included patients were recruited from nephrology clinics, our patient selection may have introduced a bias toward relatively better medical compliance among those patients with modifiable factors, including the uremic milieu and blood pressure. Second, among patients with hypertension or diabetes, patients who had presented with proteinuria before entry into the study and who had responded to medical treatment thereafter were excluded from the study unless their eGFR was $<60 \mathrm{ml} \mathrm{min}^{-1}$ per $1.73 \mathrm{~m}^{2}$. Thus, the patients with diabetes or $\mathrm{HN}$ included in the present study may have been relatively resistant to conventional therapies. This resistance may have made their outcomes relatively worse, even though we adjusted for positive findings for proteinuria. Finally, data on microalbuminuria were not available in the present study. Because the clinical significance of microalbuminuria has been well demonstrated, further study is needed to determine the effect of microalbuminuria in these patients.

In conclusion, the present study demonstrated that patients with $\mathrm{HN}$ are at increased risk of CVD events and death among non-diabetic CKD patients, which highlights the clinical significance of HN.

\section{CONFLICT OF INTEREST}

The authors declare no conflict of interest.

\section{ACKNOWLEDGEMENTS}

This study was supported by a grant from Astellas Pharm, Inc. The authors express special thanks to Mrs. Makiko Nakayama and Jun Sakaino for their assistance with the study.

Study contributors: Yuji Yamaguchi (Japanese Red Cross Sendai Hospital), Katsuya Obara (Tohoku Kosai Hospital), Isao Kurihara (Tohoku Kosai Miyagino Hospital), Yasumichi Kinoshita and Kazuto Sato (Japanese Red Cross Ishinomaki Hospital), Jin Seino (Miyagi National Hospital), Akira Sugiura and Masahiro Miyata (Osaki Citizen Hospital), Kazuhisa Takeuchi (Koujinkai Central Hemodialysis Clinic), Kenji Nakayama and Naoki Akiu (Sendai City Hospital), Tetsuya Otaka (Katta General Hospital), Osamu Hotta, Hiroo Noshiro, Kazuyuki Suzuki, Mitsuhiro Sato, Norio Ieiri, Yoshinori Tsuchiya, Kozo Sato, Tomoyoshi Kimura, and Aki Ishida (Sendai Shakaihoken Hospital), and Tasuku Nagasawa, Noriko Miyazawa, and Takuma Hosoya (Tohoku University School of Medicine). 
1 National Kidney Foundation. K/DOQI clinical practice guidelines for chronic kidney disease: evaluation, classification, and stratification. Kidney disease outcome quality initiative. Am J Kidney Dis 2002; 39(2 Suppl 2): S1-S246.

2 Manjunath G, Tighiouart H, Ibrahim H, MacLeod B, Salem DN, Griffith JL, Coresh J, Levey AS, Sarnak MJ. Level of kidney function as a risk factor for atherosclerotic cardiovascular outcomes in the community. J Am Coll Cardiol 2003; 41: 47-55.

3 Keith DS, Nichols GA, Gullion CM, Brown JB, Smith DH. Longitudinal follow-up and outcomes among a population with chronic kidney disease in a large managed care organization. Arch Intern Med 2004; 164: 659-663.

4 GO AS, Chertow GM, Fan D, McCulloch CE, Hsu C. Chronic kidney disease and the risks of death, cardiovascular events, and the hospitalization. New Engl J Med 2004; 351: $1296-1305$.

5 Ninomiya T, Kiyohara Y, Kubo M, Tanizaki Y, Doi Y, Okubo K, Wakugawa Y, Hata J, Oishi Y, Shikata K, Yonemoto K, Hirakata H, lida M. Choronic kidney disease and cardiovascular disease in a general Japanese population: the hisayama study. Kidney Int 2005; 68: 228-236.

6 Irie F, Sairenchi T, Fukasawa N, Yamagishi K, Ikehara S, Kanashiki M, Saito Y, Ota H, Nose T. The relationships of proteinuria, serum creatinine, glomerular filtration rate with cardiovascular disease mortality in Japanese general population. Kidney Int 2006; 69: 1264-1271.

7 Nakayama M, Metoki H, Terawaki H, Ohkubo T, Kikuya M, Sato T, Nakayama K, Asayama K, Inoue R, Hashimoto J, Totsune K, Hoshi H, Ito S, Imai Y. Kidney dysfunction as a risk factor for first symptomatic stroke events in a general Japanese population-the Ohasama study. Nephrol Dial Transplant 2007; 22: 1910-1915.

8 Iseki K, Ikemiya Y, Iseki C, Takishita S. Proteinuria and the risk of developing end-stage renal disease. Kidney Int 2003; 63: 1468-1474.

9 So WY, Kong AP, Ma RC, Ozaki R, Szeto CC, Chan NN, Ng V, Ho CS, Lam CW, Chow CC, Cockram CS, Chan JC, Tong PC. Glomerular filtration rate, cardiorenal end points, and all-cause mortality in type 2 diabetic patients. Diabetes Care 2006; 29: 2046-2052

10 Islam TM, Fox CS, Mann D, Muntner P. Age-related associations of hypertension and diabetes mellitus with chronic kidney disease. BMC Nephrol 2009; 10: 17.

11 Wattanakit K, Coresh J, Muntner P, Marsh J, Folsom AR. Cardiovascular risk among adults with chronic kidney disease, with or without prior myocardial infarction. J Am Coll Cardiol 2006; 48: 1183-1189. Epub 2006 Aug 28.

12 Ahmed A, Rich MW, Sanders PW, Perry GJ, Bakris GL, Zile MR, Love TE, Aban IB, Shlipak MG. Chronic kidney disease associated mortality in diastolic versus systolic heart failure: a propensity matched study. Am J Cardiol 2007; 99: 393-398.

13 Kokubo Y, Nakamura S, Okamura T, Yoshimasa Y, Makino H, Watanabe M, Higashiyama A, Kamide K, Kawanishi K, Okayama A, Kawano Y. Relationship between blood pressure category and incidence of stroke and myocardial infarction in an urban Japanese population with and without chronic kidney disease: the Suita Study. Stroke 2009; 40: 2674-2679.

14 Tsimihodimos V, Dounousi E, Siamopoulos KC. Dyslipidemia in chronic kidney disease: an approach to pathogenesis and treatment. Am J Nephrol 2008; 28: 958-973.

15 Roderick PJ, Atkins RJ, Smeeth L, Mylne A, Nitsch DD, Hubbard RB, Bulpitt CJ, Fletcher AE. CKD and mortality risk in older people: a community-based population study in the United Kingdom. Am J Kidney Dis 2009; 53: 950-960.

16 Deegens JK, Wetzels JF. Membranous nephropathy in the older adult: epidemiology, diagnosis and management. Drugs Aging 2007; 24: 717-732.

17 Glassock RJ. Prophylactic anticoagulation in nephrotic syndrome: a clinical conundrum. J Am Soc Nephrol 2007; 18: 2221-2225.

18 Roman MJ, Shanker BA, Davis A, Lockshin MD, Sammaritano L, Simantov R, Crow MK, Schwartz JE, Paget SA, Devereux RB, Salmon JE. Prevalence and correlates of accelerated atherosclerosis in systemic lupus erythematosus. N Engl J Med 2003; 349: 2399-2406.

19 Perneger TV, Klag MJ, Feldman HI, Whelton PK. Projections of hypertension-related renal disease in middle-aged residents of the United States. JAMA 1993; 269. 1272-1277
20 Lechner BL, Bockenhauer D, Iragorri S, Kennedy TL, Siegel NJ. The risk of cardiovascular disease in adults who have had childhood nephrotic syndrome. Pediatr Nephrol 2004; 19: 744-748.

21 Morgan MD, Turnbull J, Selamet U, Kaur-Hayer M, Nightingale P, Ferro CJ, Savage CO, Harper L. Increased incidence of cardiovascular events in patients with antineutrophil cytoplasmic antibody-associated vasculitides: a matched-pair cohort study. Arthritis Rheum 2009; 60: 3493-3500.

22 Nakayama M, Sato T, Sato H, Yamaguchi Y, Obara K, Kurihara I, Sato K, Hotta O, Seino J, Miyata M, Takeuchi K, Nakayama K, Matsushima M, Otaka T, Kinoshita Y, Taguma Y, Ito S. Different clinical outcomes for cardiovascular events and mortality in chronic kidney disease according to underlying renal disease: the Gonryo study. Clin Exp Nephrol 2010; 14: 333-339.

23 National Institute of Neurological Disorders and Stroke Ad Hoc Committee. Classification of cerebrovascular disease. Stroke 1990; 21: 637-676.

24 Matsuo S, Imai E, Horio M, Yasuda Y, Tomita K, Nitta K, Yamagata K, Tomino Y, Yokoyama H, Hishida A, Collaborators Developing the Japanese Equation for Estimated GFR. Revised equations for estimated GFR from serum creatinine in Japan. Am J Kidney Dis 2009; 53: 982-992.

25 Takahashi M, Fukuda Y, Iwata S. Fundamental evaluation and efficacy for protein to creatinine ratio by ATLAS kit cartridge PRO12 using automatic urine analyzer Clinitek ATLAS XL. Igaku Yakugaku 2002; 48: 727-735. (in Japanese).

26 Yamamoto S, Kon V. Mechanisms for increased cardiovascular disease in chronic kidney dysfunction. Curr Opin Nephrol Hypertens 2009; 18: 181-188.

27 Kestenbaum B, Sampson JN, Rudser KD, Patterson DJ, Seliger SL, Young B, Sherrard DJ, Andress DL. Serum phosphate levels and mortality risk among people with chronic kidney disease. J Am Soc Nephrol 2005; 16: 520-528; e-pub ahead of print 22 December 2004

28 Honda H, Qureshi AR, Heimbürger O, Barany P, Wang K, Pecoits-Filho R, Stenvinkel P, Lindholm B. Serum albumin, C-reactive protein, interleukin 6 , and fetuin a as predictors of malnutrition, cardiovascular disease, and mortality in patients with ESRD. Am J Kidney Dis 2006; 47: 139-148.

29 Chan DT, Irish AB, Dogra GK, Watts GF. Dyslipidaemia and cardiorenal disease: mechanisms, therapeutic opportunities and clinical trials. Atherosclerosis 2008; 196: 823-834. e-pub ahead of print 6 March 2007.

30 Kazory A, Ross EA. Anemia: the point of convergence or divergence for kidney disease and heart failure? J Am Coll Cardiol 2009; 53: 639-647.

31 Himmelfarb J, Stenvinkel P, Ikizler TA, Hakim RM. The elephant in uremia: oxidant stress as a unifying concept of cardiovascular disease in uremia. Kidney Int 2002; 62: 1524-1538.

32 Vaziri ND, Rodriguez-Iturbe B. Mechanisms of disease: oxidative stress and inflammation in the pathogenesis of hypertension. Nat Clin Pract 2006; 2: 582-593.

33 Ravani P, Tripepi G, Malberti F, Testa S, Mallamaci F, Zoccali C. Asymmetrical dimethylarginine predicts progression to dialysis and death in patients with chronic kidney disease: a competing risks modeling approach. J Am Soc Nephrol 2005; 16 : 2449-2455; e-pub ahead of print 8 June 2005.

34 Barreto FC, Barreto DV, Liabeuf S, Meert N, Glorieux G, Temmar M, Choukroun G, Vanholder R, European Uremic Toxin Work Group (EUTox). Serum indoxyl sulfate is associated with vascular disease and mortality in chronic kidney disease patients. Clin J Am Soc Nephrol 2009; 4: 1551-1558.

35 Cheng J, Zhang X, Zhang W, He Q, Tao X, Chen J. Efficacy and safety of glucocorticoids therapy for IgA nephropathy: a meta-analysis of randomized controlled trials. $\mathrm{Am} \mathrm{J}$ Nephrol 2009; 30: 315-322.

36 Wilson AC, Mitsnefes MM. Cardiovascular disease in CKD in children: update on risk factors, risk assessment, and management. Am J Kidney Dis 2009; 54: 345-360.

37 Lilien MR, Groothoff JW. Cardiovascular disease in children with CKD or ESRD. Nat Rev Nephrol 2009; 5: 229-235.

38 Peritoneal Dialysis Adequacy Work Group. Clinical practice guidelines and clinical recommendations 2006 update Clinical practice guidelines for peritoneal dialysis adequacy. Am J Kidney Dis 2006; 48(Suppl 1): S98-S129.

39 Dombros N, Dratwa M, Feriani M, Gokal R, Heimbürger O, Krediet R, Plum J, Rodrigues A, Selgas R, Struijk D, Verger C, EBPG Expert Group on Peritoneal Dialysis. European best practice guidelines for peritoneal dialysis 2 . The initiation of dialysis. Nephrol Dial Transplant 2005; 20(Suppl 9): ix3-ix7. 